\title{
Bovhyaluronidaze azoximer as an antimicrobial alternative in companion animals
}

\author{
Anna Nazarova $^{1 *}$, Boris Semenov ${ }^{1}$, Anna Kozitcyna ${ }^{1}$, Veronika Guseva ${ }^{1}$, Tatiana Kuznetsova $^{1}$, and Vladimir Videnin ${ }^{1}$ \\ ${ }^{1}$ Saint-Petersburg State University of Veterinary Medicine, Saint Petersburg, Russia
}

\begin{abstract}
Antimicrobial resistance formation in various bacteria is a serious biosafety threat. In this regard, the important issue in medical and veterinary fields is not only the rational use of antibacterial drugs, but also the search for ways to significantly reduce the need for antibiotics. Three studies designed to research into these challenges are described. The results indicated that (1) the bacteriuria presences in $62.4+/-4.1 \%$ of urine samples obtained from companion animals (the microflora count in urine samples is estimated from '++' to 'Entire Field-of-View'); (2) $16.3+/-4.0 \%$ of samples from companion animals showed bacteria with low antibiotics sensitivity, and $8.1+/-3.0 \%$ of samples contained bacteria resistant to 30 antibiotics included in the susceptibility test; (3) the Bovhyaluronidaze azoximer reduce antimicrobial therapy duration statistically significant $(\mathrm{p}=0.0089)$. The studies were conducted at the facilities of St. Petersburg State University of Veterinary Medicine and Bars veterinary clinic chain in St. Petersburg, Russia. A randomized clinical trial of Bovhyaluronidaze azoximer effect on course of disease and treatment duration of acute and chronic prostatitis and urocystitis, including complicated by urolithiasis, was conducted in 54 dogs. Animals were randomly assigned to intervention $(n=34)$ and control $(n=20)$ groups. Animals of both groups received the antimicrobial therapy. Interventional group patients additionally received Bovhyaluronidaze azoximer in the form of rectal suppositories. The antimicrobial therapy mean duration in intervention group animals took $14.88+/-4.98$ days, in control group animals $-18.65+/-5.40$ days. The study findings imply that bacteriuria is widely prevalent in companion animals and antimicrobial resistance is increasing issue in veterinary medicine. Bovhyaluronidaze azoximer contributes to microcirculation restore and increases the bioavailability of antibacterial drugs at the site of infection. This makes its relevant for use in the complex treatment for septic and inflammatory processes. This allows to reduce the antimicrobial course duration (and completely decline antibiotic therapy in some cases) and prevent the formation of chronic inflammation and infection sites.
\end{abstract}

\section{Introduction}

Currently, due to the fast rate of antimicrobial resistance widespread in various bacteria, an important biosafety issue in both medical and veterinary fields is not only the rational use of antimicrobial agents, but also the search for medications that can significantly reduce the need for antibiotics. An important component of antimicrobial resistance problem is the formation of bacterial biofilms. Bacteria living in a biofilm can be protected from both antimicrobial agents and immune system [1].

As an alternative to antibiotics, research is being carried out on the possibility of using pharmaceutical preparations based on silver such as Silver Chloride Gel [2] and Argentum Quartz (R) [3], probiotics such as Lactobacillus plantarum [4], Curcumin oile [5] and new types of bandages, for example the Lawsonia Inermisgelatin-starch nano-fibrous bandage [6]. However, these methods are only for topical use and do not have a systemic effect, so they does not act upon the site of chronic infection (which often forms after the biofilm formation), and also does not affect the immune system.
Urinary tract infections are the common reason for the antimicrobial use in veterinary medicine, which promotes antimicrobial resistance in urological patients [7, 8].

The Department of Obstetrics and Operative Surgery of the St. Petersburg State University of Veterinary Medicine underwent studies of Bovhyaluronidaze azoximer (lat.: Bovhyaluronidazum azoximerum), a stabilized form of enzyme hyaluronidase (hyaluronidase conjugate with a high-molecular derivative of poly-1,4ethylene piperazine $\mathrm{N}$ oxide). Development and production - NPO "Petrovax Pharm", Russia.

Studies of Bovhyaluronidaze azoximer use are carried out in the following areas:

- prevention of postoperative complications in urological patients who underwent bladder and urethra surgery;

- treatment of animals with purulent-inflammatory processes in various sites;

- treatment of animals with chronic urocystitis, including complicated by urolithiasis, as well as with chronic diseases of the prostatic gland.

\footnotetext{
* Corresponding author: anna.v.nazarova@ mail.ru
} 


\section{Experimental}

The studies were conducted at the facilities of Bars veterinary clinic chain in the city of St. Petersburg, Russia, and St. Petersburg State University of Veterinary Medicine. Laboratory tests, including urinalysis, urine bacterial culture and antimicrobial susceptibility tests, were performed in the Bars-Diagnostics laboratory, St. Petersburg.

To assess the prevalence rate of bacteriuria the microflora count in urine samples from 141 cats was evaluated.

To assess the prevalence of antimicrobial resistance, the results of a disc-diffusion antibiotic sensitivity test were analyzed in 86 samples (57 dogs and 29 cats). 30 antibacterial agents were included in the tests: Amoxicillin, Ampicillin, Azithromycin, Aztreonam, Bacitracin, Cefazolin, Cefotaxime, Ceftriaxone, Clarithromycin, Clindamycin, Doxycycline, Enrofloxacin, Erythromycin, Furadonin, Furagin, Furazolidone, Imipenem, Levofloxacin, Lincomycin, Lomefloxacin, Nalidixic acid, Norfloxacin, Novobiocin, Ofloxacin, Oxacillin, Polymyxin B, Tetracycline, Trimethoprim / Sulfamethoxazole, Tylosin, Vancomycin.

Study of Bovhyaluronidaze azoximer effect on the course of disease and treatment duration of acute and chronic prostatitis and urocystitis, including complicated by urolithiasis, was conducted in 54 animals.

In this randomized clinical trial 54 dogs (Canis lupus familiaris) with urogenital system diseases were randomly assigned to intervention $(n=34)$ and control $(n=20)$ groups. The intervention group included 22 males and 12 females. The control group included 15 males and 5 females.

The mean age of the dogs in the intervention group was $6.24 \pm 2.83$ years, in the control group $-6.80 \pm 3.69$ years. The mean weight of the dogs was $7.62 \pm 8.72 \mathrm{~kg}$ and 12.09 $\pm 9.71 \mathrm{~kg}$ in the intervention and control groups respectively (Standard Deviation (SD) is indicated).

Prostatitis was diagnosed in 19 dogs (10 dogs in the intervention group and 9 dogs in the control group). Bacterial urocystitis was diagnosed in 24 dogs (16 dogs in the intervention group and 8 dogs in the control group). Bacterial urocystitis complicated by urolithiasis was diagnosed in 9 dogs ( 7 dogs in the intervention group and 2 dogs in the control group). One dog in the experimental group had a penile trauma and $1 \mathrm{dog}$ in the control group had acute urinary retention.

Clinical examination of all animals was carried out in accordance with GOST R 58090-2018 "Clinical examination of unproductive animals" [9]. The diagnosis was established on the basis of anamnesis, physical examination, the results of the urinalysis and visual diagnostic data (ultrasound and x-ray studies).

The animals of both groups receiver antibiotic therapy in accordance with generally accepted recommendations for the use of drugs and in accordance with the results of the antibiotic susceptibility test (in those cases when these tests were performed). Patients of the interventional group additionally received Bovhyaluronidaze azoximer in the form of rectal suppositories.

The effectiveness of antibiotic therapy was assessed by the presence of microflora in the controlled urine samples.
We performed the statistical analysis in Microsoft Excel 2016 and BioStat, AnalystSoft Inc., version 7.

The statistical significance (alpha level) adopted in this study is $5 \%(\mathrm{p}=0.05)$.

To compare the treatment duration in the interventional and control groups the Mann-Whitney U Test was used. If the significance level $\mathrm{p}$ obtained by applying the criterion was less than 0.05 , the differences between the groups were recognized as statistically significant.

\section{Results and discussion}

\section{Bacteriuria prevalence}

Table 1 presents the results of a statistical analysis of 141 urine samples obtained from animals admitted with symptoms of urinary tract distress. Only in $37.6 \pm 4.1 \%$ of the samples were detected no microflora or the count was insignificant ('+') (hereinafter, Standard Error of Mean (SEM) is indicated). In $62.4 \pm 4.1 \%$ of the samples, the microflora count was estimated from ' ++ ' to 'Entire Fieldof-View'.

Thus, it is established that in most cases in animals with urinary tract diseases antimicrobial therapy should be part of a complex therapy. However, errors such as unreasonable prescription of antibiotics for the prevention of bacterial infections, improper selection of dose or administration frequency and course duration can reduce the effectiveness of antimicrobial therapy, as well as lead to a variety of side effects, such as the disease transition into a chronic form and the formation of antimicrobial resistance bacteria strains.

Antimicrobial resistance prevalence

In veterinary practice, antibiotic susceptibility test is not carried out routinely during the initial treatment in the clinic. If a bacterial cause is suspected, antimicrobial agent is empirically prescribed. Often, with the ineffectiveness of the first antibacterial agent, an antibiotic change is also carried out empirically, without an antibiotic susceptibility test.

Table1. Microflora in the urinalysis samples

\begin{tabular}{|c|c|c|}
\hline \multirow{2}{*}{$\begin{array}{c}\text { The count of } \\
\text { microflora }^{\text {a }}\end{array}$} & \multicolumn{2}{|c|}{$\begin{array}{c}\text { Percentage of the urinalysis } \\
\text { samples (\%) }\end{array}$} \\
\cline { 2 - 3 } & Percentage & \pm SEM \\
\hline- & 1.4 & \pm 1.0 \\
\hline+ & 36.2 & \pm 4.1 \\
\hline++ & 19.1 & \pm 3.3 \\
\hline+++ & 5.7 & \pm 2.0 \\
\hline++++ & 2.8 & \pm 1.4 \\
\hline $\begin{array}{c}\text { Entire Field-of- } \\
\text { View }\end{array}$ & 34.8 & \pm 4.0 \\
\hline
\end{tabular}

As a result, by the time the animal owners agreeing to conduct antibiotic susceptibility test, there is often an association of microorganisms in the infection site, and/or microorganisms that have developed resistance to a large number of antibiotics.

Eighty sex results of a disc-diffusion antibiotic sensitivity tests were analyzed. The animals mean age from which the samples were taken was $6.6 \pm 4.0$ years ( $\pm S D$ is indicated). 
Of these, 44 samples $(51.2 \%)$ were the urine samples, 30 samples $(34.9 \%)$ were the materials from the ears, 6 samples $(7.0 \%)$ were the materials from the eyes, and 3 samples (3.5\% each) were the materials from the larynx and skin.

A culture of Staphylococcus aureus was found in $97.7 \pm 1.6 \%$ (hereinafter $\pm S E M$ is indicated) of the samples, and Escherichia coli in $37.2 \pm 5.2 \%$ of the samples. In $44.2 \pm 5.4 \%$ of the samples, an association of microorganisms was found. Oxacillin resistance was established in microorganisms from $66.3 \pm 5.1 \%$ of samples.

There were detected low antibiotic sensitivity for bacteria from $16.3 \pm 4.0 \%$ of the samples (for 30 antibacterial agents included in the tests), and bacteria from $8.1 \pm 3.0 \%$ of the samples had antibiotic resistance to all 30 antibiotics included in the tests (all 30 antimicrobial agents were ineffective).

In the 44 urine samples culture of Staphylococcus aureus was found in $95.5 \pm 3.2 \%$ (hereinafter $\pm S E M$ is indicated) of the urine samples, and Escherichia coli in $52.3 \pm 7.6 \%$ of the urine samples. In $52.3 \pm 7.6 \%$ of the urine samples, an association of microorganisms was found. Oxacillin resistance was established in microorganisms from $75.0 \pm 6.6 \%$ of urine samples.

There were detected low antibiotic sensitivity for bacteria from $18.2 \pm 5.9 \%$ of the samples (for 30 antibacterial agents included in the tests), and bacteria from $15.9 \pm 5.6 \%$ of the urine samples had antibiotic resistance to all 30 antibiotics included in the discdiffusion antibiotic sensitivity tests (all 30 antimicrobial agents were ineffective).

Therefore, it is established that urine samples bacterial culture possess higher antibacterial resistance in comparison with samples from other sites.

Therapy of patients with antimicrobial resistant microflora requires not only the selection of antimicrobial agents and their use in an adequate dose and sufficient course duration, but also the bioavailability of the antibiotic in the inflammation site and prevention chronic inflammation and infection sites formation.

Chronic urocystitis and prostatitis treatment duration reduction

In the treatment of chronic urocystitis and prostatitis in intervention group animals the Bovhyaluronidaze azoximer in the form of suppositories was administered rectally in addition to antimicrobial therapy.

For dogs of the intervention group weighing up to 10 $\mathrm{kg}$, the Bovhyaluronidaze azoximer was administered at a dose of $750 \mathrm{IU}$ per animal, for dogs weighing up to $30 \mathrm{~kg}$ the Bovhyaluronidaze azoximer was administered at a dose of 1,500 IU per animal, and for dogs weighing more than $30 \mathrm{~kg}-3,000 \mathrm{IU}$ Bovhyaluronidaze azoximer per animal. Bovhyaluronidaze azoximer suppositories were administered rectally $\mathrm{q} 48 \mathrm{~h}, 10$ administrations, and then q72h, 10 administrations.

One animal experienced bleeding after the drug administration (further use was discontinued). In 3 animals, dose-related side effects were observed in the form of lethargy and appetite loss. After dose reducing to $500 \mathrm{IU}$ of Bovhyaluronidaze azoximer per animal, the side effects ceased.
The mean antimicrobial therapy duration in animals of the intervention group was $14.88 \pm 4.98$ days $( \pm S D$ is indicated).

The mean antimicrobial therapy duration in animals of the control group was $18.65 \pm 5.40$ days $( \pm S D$ is indicated).

When comparing the results obtained in the intervention and control groups using the Mann-Whitney $U$ Test, the p-value is 0.0089 , which is much less than the alpha level adopted in our study $(\mathrm{p}=0.05)$ and allows us to conclude that the antimicrobial therapy length reduction in animals of the intervention group is statistically significant.

A summary diagram of the length of treatment for 3 diseases is presented in Fig. 1.

Mechanism of action of Bovhyaluronidaze azoximer on the course of inflammatory processes

Urgent conditions such as acute or recurrent urinary retention, protracted chronic urinary retention caused by obstruction and/or urethral stenosis, injuries and ruptures of the urethra require surgical interventions such as cystocentesis, bladder catheterization, cystotomy, perineal urethrostomy in order to save animal's life. In this regard, the goal is to minimize the complications that may be caused by such interventions.

The most common complications of perineal urethrostomy in the short term are: hematomas, subcutaneous urine infiltration in the area of urine leakage between the urethra mucous membrane and the skin, repeated urethra obstruction with blood clots and/or due to edema of urethra mucous membrane and periurethral tissue, excoriation of the perineum and suture failure [10].

\section{Length of treatment}

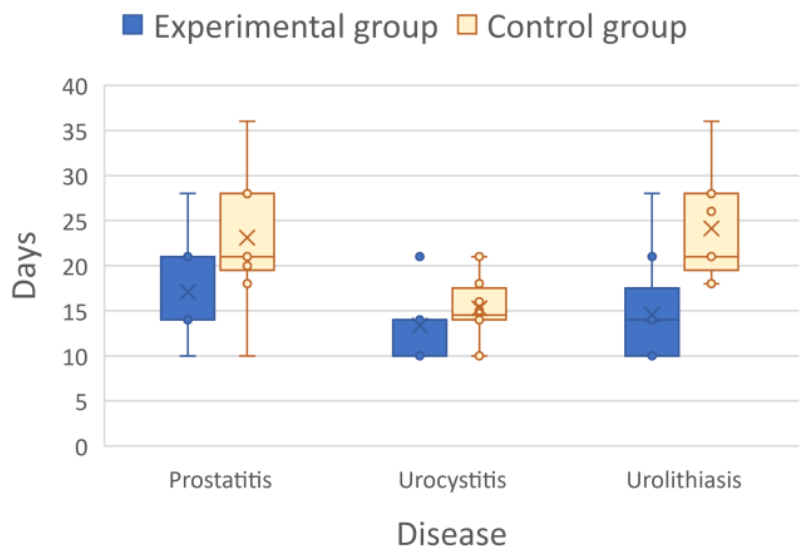

Fig. 1 Confidence intervals for 3 diseases in the intervention and controlgroups

In long term, complications of perineal urethrostomy may include chronic dermatitis of the skin fold, the bladder atony, urinary incontinence, and urethral stricture [11]. Urethral strictures and urethrostomy overgrowth, according to different authors, range from $12 \%$ [12] to $35 \%$ [13]. Recurrent urinary tract infection is observed in more than $30 \%$ of cats undergoing perineal urethrostomy and in more than $60 \%$ of cats undergoing prepubic urethrostomy $[14,15]$. Complications such as postoperative urinary tract infections are also observed 
after cystotomy [16]. In this regard, antimicrobial agents are often used during postoperative period.

Acute inflammatory process is accompanied by the edema formation. Edema disrupts adequate blood supply, reduces the bioavailability of antibacterial drugs, as a result tissue healing slows down, and chronic inflammation and infection site is created. The chronic inflammatory process that forms as a result of prolonged edema in $100 \%$ of cases is characterized by the formation of scar (granulation) tissue [17].

The degree of edema (including edema of periurethral tissues after perineal urethrostomy) significantly affects the development of postoperative complications. The chronic inflammatory process caused by prolonged edema leads to fibrotic changes in the periurethral tissues, increases the rigidity of the urethra and bladder, causing relapses of acute urinary retention. Also, the formation of a site of chronic infection, incomplete emptying of the bladder, urine stagnation and relapses of acute urinary retention lead, in turn, to the formation of antimicrobial resistant bacteria strains, including oxacillin-resistant strains.

As a result, the following tasks are set in the course of inflammatory process treatment:

-reduce swelling and tissue inflammation,

-restore microcirculation,

-ensure higher antibiotics bioavailability in the infectious site,

-reduce the synthesis of connective tissue components, -prevent the fibrotic changes formation in tissues.

To restore microcirculation and normalize connective tissue processes in the inflammation area, the Bovhyaluronidaze azoximer (lat.: Bovhyaluronidazum azoximerum) was included in the complex therapy of intervention groups animals. The Bovhyaluronidaze azoximer contains an immobilized hyaluronidase hyaluronidase conjugate with a high-molecular carrier. Hyaluronidase hydrolyzes the hyaluronan (high-molecular hyaluronic acid) to tetrasaccharides [18], which reduces the viscosity of the connective tissue extracellular matrix, thereby increasing the permeability of tissues, facilitating the fluids movement in interstitial spaces and preventing pathological growth of connective tissue.

In addition, the high-molecular carrier, being an activated derivative of poly-1,4-ethylene piperazine Noxide, possesses its own pharmacological activity and regulates the synthesis of inflammatory mediators interleukin-1 (IL 1) and tumor necrosis factor-alpha (TNF$\alpha$ ), increases the humoral immune response and resistance to infections [19].

In our study, the proportion of animals in the intervention group with postoperative complications was 0.10 . In the control group, the proportion of animals with postoperative complications was 0.55 [20].

Using the Pearson's Chi Squared Test $\left(\chi^{2}\right)$ to compare the frequency of urethral and bladder surgery complications with Bovhyaluronidaze azoximer during postoperative period, it was found that there is a statistically significant relationship between the use of Bovhyaluronidaze azoximer and the frequency of postoperative complications, regardless of the type of surgery.
Considering that a preoperative urinary tract infection is associated with postoperative complications development [21], it is reasonable to use Bovhyaluronidaze azoximer during the animal preoperative preparation in order to shorten the duration of the antimicrobial course and reduce the inflammatory process severity.

Due to the anti-edematous and immunostimulating action of Bovhyaluronidaze azoximer as part of purulentinflammatory processes complex therapy, the median wound cleansing from purulonecrotic masses duration is reduced by 2.29 days. The median duration of edema reduction is reduced by 1.57 days and the median number of necessary wound management is reduced by 5.00 manipulations [22].

When comparing the results obtained in the intervention and control groups using the Mann-Whitney $U$ Test, the p-value is significantly lower than the significance level adopted in our study $(\mathrm{p}=0.05)$, which indicates that the differences are statistically significant.

Currently, we continue research in this direction and form an intervention group of animals in which systemic antibacterial therapy is not used as a part of purulentinflammatory processes treatment, and topical treatment is combined with the Bovhyaluronidaze azoximer in the form of rectal suppositories.

Fig. 2 shows an abscess of the left perianal sinus in a 3-year-old male Chihuahua, admitted to the clinic with complaints of restlessness and severe pain in the perianal region.

It was decided not to use a systemic antimicrobial in this animal. The course of Bovhyaluronidaze azoximer was reduced to 4 administrations, since by the 4th day of use the edema had completely subsided; the abscess cavity was completely cleansed. The abscess cavity was completely healed by the 7th day of treatment (Fig. 3).

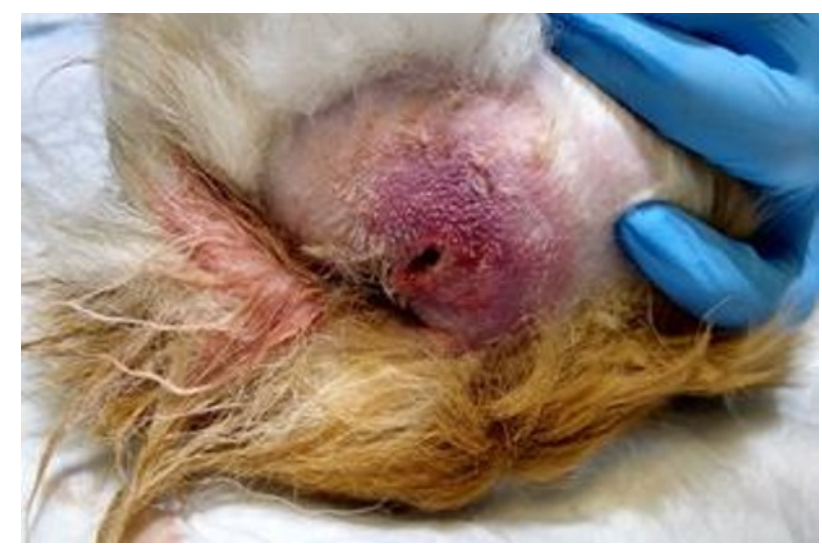

Fig.2 Abscess of the left perianal sinus (a lancing of abscess was performed)

Because the hyaluronidase in Bovhyaluronidaze azoximer is covalently attached to a high-molecular carrier, the therapeutic doses are small enough to avoid allergic reactions. This, in turn, makes Bovhyaluronidaze azoximer the first-choice even if the patient is in a serious condition at the time of the operation, for example, with a urethral injury before surgery, extensive hematomas, infiltration of periurethral and perirectal tissues, 
subcutaneous tissue, and intermuscular spaces of the pelvic muscles with urine [23].

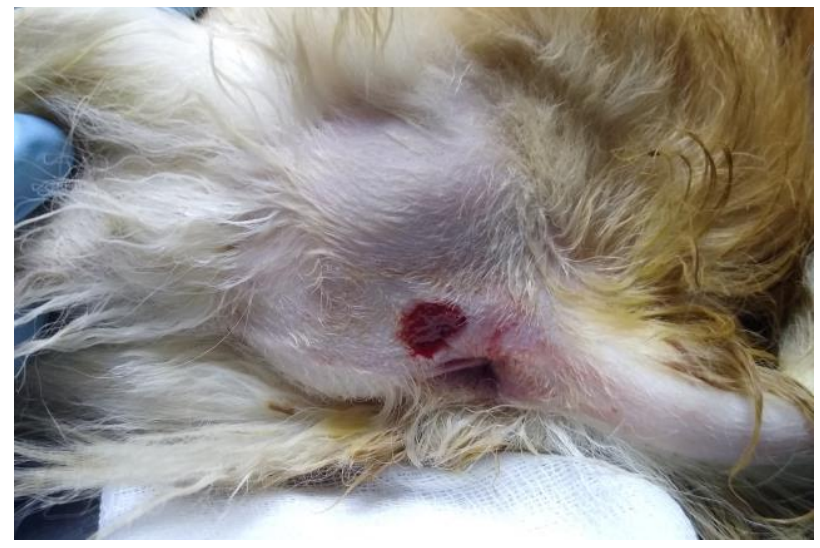

Fig 3. The same animal as in Fig. 2, on the 7th day of treatment

\section{Conclusion}

This study showed that Bovhyaluronidaze azoximer has an anti-inflammatory effect, reduces the severity of edema and pain reactions and is able to prevent reactive growth of connective tissue as well as fibrosis development and complications of surgical intervention both in the short and long term.

The use of the Bovhyaluronidaze azoximer during the pre- and postoperative period of operations on the urethra and bladder can quickly reduce edema and inflammation of the surgical wound tissues and tissues around the urethrostomy. It also prevents coarse scarring of the postoperative suture between the urethra mucous membrane and the skin, which leads to the urethrostoma closing and/or urethral strictures. As a result, the use of the Bovhyaluronidaze azoximer statistically significantly reduces the incidence of postoperative complications.

The results of our studies indicate that Bovhyaluronidaze azoximer statistically significantly reduces duration of treatment, accelerates the wounds and cavities purification from purulent-necrotic contents, significantly reduces edema and tissue inflammation, helps restore microcirculation, and increases the bioavailability of antibacterial agents in the infection site. This allows reduce the duration of antibiotic courses (and in some cases to completely abandon antibiotic therapy) and prevent the formation of chronic infection site.

Due to the covalent binding of hyaluronidase to a highmolecular carrier Bovhyaluronidaze azoximer has a prolonged action and resistance to denaturing agents which makes it convenient to use (which, in turn, increases the compliance of animal owners), and the likelihood of allergic reactions is reduced because of enzyme low therapeutic doses. In addition, as a result of hyaluronidase conjugation, it became possible to use the Bovhyaluronidaze azoximer in the acute phase of the inflammatory process when antibiotic therapy is conducted. Consequently, the bioavailability of antibacterial drugs increased and that also makes it possible to reduce the antimicrobial courses duration without loss of therapy effects.

\section{References}

1. L. K.Vestby, vol. Gronseth, R. Simm, and L. L. Nesse, Antibiotics-Basel, 9, 29 (2020).

2. C. O'Meara, B. L. Boyanton, D. Spurlin, and C. F. Carpenter, Infectious Diseases in Clinical Practice, 24, 112-114 (2016).

3. V. D. Palumbo, P. Tralongo, B. Di Trapani, F. Carini, and G. Tomasello, Clinica Terapeutica, 170, E328-E331 (2019).

4. C. Nhan, A. Bezdjian, S. Saha, S. Prakash, L. H. P. Nguyen, and S. J. Daniel, Journal of Otolaryngology-Head \& Neck Surgery, 46 (2017).

5. M. E. Ahmed, S. H Seddiq., R. Q. Basi, G. A. Abdulhasan, and A. Q. Al-Awadi, Research Journal of Pharmaceutical Biological and Chemical Sciences, 9, 1186-1196 (2018).

6. Z. Hadisi, J. Nourmohammadi, and S. M. Nassiri, Int. J. Biol. Macromol., 107, 2008-2019 (2018).

7. R. Dorsch, C. von Vopelius-Feldt, G. Wolf, R. S. Mueller, R. K. Straubinger, and K. Hartmann, Tieraerztliche Praxis Ausgabe Kleintiere Heimtiere, 44 , 227-236 (2016).

8. S. Teichmann-Knorrn and R. Dorsch, Tieraerztliche Praxis Ausgabe Kleintiere Heimtiere, 46, 247-257 (2018).

9. GOST R 58090-2018 "Clinical examination of unproductive animals. General requirements", 2018.

10. Daniel D. Smeak, Proceedings of the North American veterinary conference, 1452-1454, 2006.

11. Clara S.S. Goh and Howard B. Seim, Today's Veterinary Practice, 43-49 (2014).

12. Karen M. Tobias, , NAVC clinician's brief, 1922 (2007).

13. B. S. Semenov, and A. V. Nazarova, "Perineal urethrostomy male cats: Pros and Cons", International bulletin of Veterinary Medicine, 2, 130-135 (2018).

14. M. T. Watson, R. Y. Roca, A. H. Breiteneicher, and R. H. Kalis, Journal of Feline Medicine and Surgery, 22, 399-403 (2020).

15. R. P. Sousa, D. C. S. Nunes-Pinheiro, K. O. Sampaio, E. C. B. da Silva, G. Cavalcanti, and M. da Cunha, Journal of Feline Medicine and Surgery, in press.

16. Z. Nikousefat, M. Hashemnia, M. Javdani, and A. Ghashghaii, Veterinary Research Forum, 9, 199-203 (2018).

17. A. V. Zaitsev, Effective Pharmacotherapy, 2, 50-56 (2018).

18. R. U. Habriyev, N. O. Kamayev, T. I. Danilova, and E. G. Kakhoyan, Biomedical Chemistry, 62, 82-88 (2016). 
19. Yu. G. Alyayev, Ye. V. Lartsova, and L. G. Spivak, Effective Pharmacotherapy, 49, 4-8, 2015.

20. A. A. Stekolnikov, A. V. Nazarova, B. S. Semenov, and T. Sh. Kuznetsova. International bulletin of Veterinary Medicine, 4, 158-165, 2019.

21. D. L. Frem, H. A. Hottinger, S. L. Hunter, and N. J. Trout, Javma-Journal of the American Veterinary Medical Association, 251, 935-940 (2017).
22. A. V. Nazarova, B. S. Semenov, A. A. Stekolnikov, and T. Sh. Kuznetsova, "The use of Bovhyaluronidaze azoximer preparation in the treatment of suppurative wounds", BIO Web of Conferences,

23. A. V. Nazarova, B. S. Semenov, A. V. Bokarev, and A. Yu. Nechaev, Collection of scientific papers of the Ninth international interuniversity conference on clinical veterinary medicine, 97110 (2019). 